\title{
Sound transmission loss of porous materials in ducts with embedded periodic scatterers
}

Dibya P. Jena, and Xiaojun Qiu

Citation: The Journal of the Acoustical Society of America 147, 978 (2020); doi: 10.1121/10.0000650

View online: https://doi.org/10.1121/10.0000650

View Table of Contents: https://asa.scitation.org/toc/jas/147/2

Published by the Acoustical Society of America

\section{ARTICLES YOU MAY BE INTERESTED IN}

Broadband low frequency sound absorption using a monostable acoustic metamaterial

The Journal of the Acoustical Society of America 147, EL113 (2020); https://doi.org/10.1121/10.0000714

Acoustic metamaterial capsule for reduction of stage machinery noise

The Journal of the Acoustical Society of America 147, 1491 (2020); https://doi.org/10.1121/10.0000857

Sound transmission loss of multi-layered infinite micro-perforated plates

The Journal of the Acoustical Society of America 147, 508 (2020); https://doi.org/10.1121/10.0000600

Green's function approach for the transmission loss of concentrically multi-layered circular dissipative chamber The Journal of the Acoustical Society of America 147, 867 (2020); https://doi.org/10.1121/10.0000675

Estimation of angle-dependent absorption coefficients from spatially distributed in situ measurements The Journal of the Acoustical Society of America 147, EL119 (2020); https://doi.org/10.1121/10.0000716

Acoustic metasurface-based perfect absorber with deep subwavelength thickness Applied Physics Letters 108, 063502 (2016); https://doi.org/10.1063/1.4941338

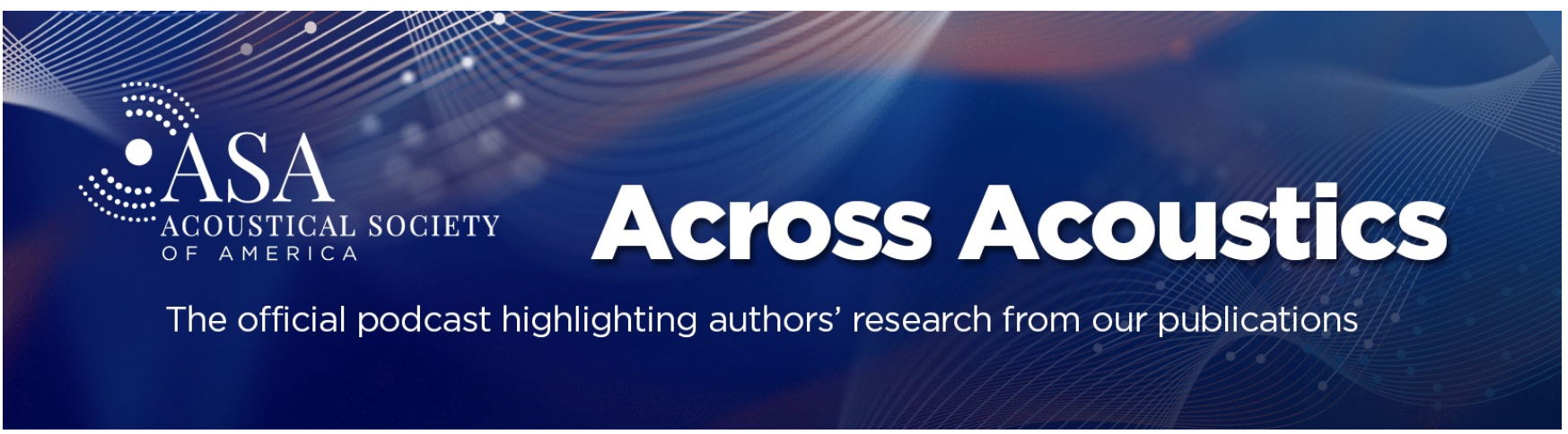




\title{
Sound transmission loss of porous materials in ducts with embedded periodic scatterers
}

\author{
Dibya P. Jena ${ }^{a)}$ and Xiaojun Qiu ${ }^{\text {b) }}$ \\ Centre for Audio, Acoustics and Vibration, Faculty of Engineering and Information Technology, University of Technology Sydney, \\ Sydney, Australia
}

\begin{abstract}
:
Porous materials that are commonly used for sound absorption have poor sound insulation capability. In this paper, rigid scatterers are installed periodically inside porous materials to improve their transmission loss (TL) with the Bragg diffraction. The Delany-Bazley impedance model is used to model the porous material and the transfer matrix method is adopted to calculate the TL of the mixed structure in a duct. Simulation results with a different number of scatterers and porous materials with different airflow resistivity show that the TL of porous materials can be increased significantly with periodically arranged scatterers. The decoupled analysis reveals that the TL of the mixed structure is larger than the sum of the TL of individual components in most frequency bands, except that around the first Bragg resonance frequency. C 2020 Acoustical Society of America. https://doi.org/10.1121/10.0000650

(Received 30 July 2019; revised 13 January 2020; accepted 13 January 2020; published online 10 February 2020)

[Editor: Michael J. White]

Pages: 978-983
\end{abstract}

\section{INTRODUCTION}

It is well known that porous materials generally have good sound absorption but poor sound insulation capabilities (Allard and Atalla, 2009; Pieren and Heutschi, 2015). For attaining high sound transmission loss (TL), multi-layered porous materials can be used (Utley and Mulholland, 1968; Takahashi, 1989). It is also known that periodic scatterers or sonic crystals can have large TL in some specific frequency bands due to the Bragg diffraction (Sanchez-Perez et al., 2002; Gupta et al., 2012). This paper investigates sound TL of porous materials in a duct with embedded periodic scatterers.

A significant amount of research has been carried out to increase the sound absorption of porous materials by using periodic arrangements. Olny and Boutin (2003) investigated the dual porosity material at a microscopic level to control the absorption coefficient in the desired frequency band, where the periodic perforated microporous material was considered by using the homogenization technique and was applied to the triple-scales heterogeneous materials. Roh and Yoon (2004) investigated periodic cylindrical pores in a porous material at the microscopic level, and thermal and viscous losses in porous material pores were considered. Lagarrigue et al. (2013) proposed a tunable acoustic absorber by integrating rigid inclusions such as slotted metallic cylinders in porous material to achieve high sound absorption in the low frequency range. Meta-porous materials with various shapes of inclusions such as cube, cylinder, sphere, cone, and ring torus were studied by Groby et al. (2014), and they found that the sound absorption in the low

\footnotetext{
${ }^{a)}$ Current address: Department of Industrial Design, National Institute of Technology Rourkela 769008, India. Electronic mail: dpj10@iitbbs.ac.in ${ }^{b)}$ ORCID: 0000-0002-5181-1220.
}

frequency range only hinges on the filling fraction of the inclusions instead of the shape. The difference in this paper is that the sound TLs of porous materials with embedded periodic scatterers are studied in a duct.

Sound TL of porous materials can be improved with periodic arrangements. Leclaire et al. (2015) investigated the air-saturated porous media, which has periodic lateral air cavities in a duct, and found that the sound absorption and transmission coefficients were influenced significantly by the length of periodic air cavities. Doutres et al. (2015) explored periodic inclusions of spherical Helmholtz resonators inside porous material, and they found that the acoustic performance is affected by the resonant material and the orientation of the resonator neck. The periodic arrangements considered in the above-mentioned studies are not along the direction of sound propagation, which is different from the research in this paper.

Periodic scatterers, also being called sonic crystals, can increase sound attention significantly in their Bragg resonance zone, and the attenuation depends upon the number of rows of periodic arrangement (Gupta et al., 2012; SanchezPerez et al., 2002). Researchers have proposed many hybrid configurations to increase the bandwidth such as using scatterers with porous cores or multi resonant scatterers (Sánchez-Dehesa et al., 2011; Aa and Forssén, 2014).

For example, Gulia and Gupta (2018) investigated a hybrid configuration of scatterers and porous material. They examined various configurations such as the sonic crystal bounded by two acoustic panels, the sonic crystal inside a glass wool, and the sonic crystal inside a glass wool bounded by acoustic panels. They observed high TL around Bragg frequencies but without giving much detailed analyses. In the present research, the porous medium embedded with periodic scatterers in a duct is investigated in a wider frequency range above the Bragg resonance frequency, and 
the coupled behaviour of the periodic scatterers and porous material in the frequency passband above the first Bragg resonance is discussed.

The transfer matrix method is suitable for calculating the propagation of multi-layered homogeneous and isotropic porous materials in a duct below the duct cut-off frequency (Song and Bolton, 2000; Munjal, 2014), while numerical models can be used for simulations in the whole band with complex geometrical configurations and inclusions (Kang and Bolton, 1996; Panneton and Atalla, 1996).

The motivation of the present work is to explore the mechanisms of combining porous materials with rigid scatterers to increase the TL of the mixed structures. The potential application scenarios are the situations where the completed enclosures or blocking cannot be used for noise reduction. Recently, for noise controlling in similar situations, Tang (2018) proposed an array of sound scatterers into the window cavity of a plenum window and achieved about 4-5 dB improvement in TL of the window. Sometimes, acoustic foams are required to be inserted into the air path to filter out dust and harmful particles from traffic and the outside environment, which can further increase the noise reduction performance of the window at the cost of some loss of air exchange rate. The target frequency range of the present work is for the middle to high frequency range of noise control from 2 to $6 \mathrm{kHz}$. Nevertheless, this method is not effective for low frequency noise control due to the low acoustic transmission coefficients of the porous materials and large wavelength for less scattering in the low frequency range.

In this paper, the numerical simulation results are verified by the analytical results based on the transfer matrix method first, and then the numerical simulations are used to calculate the TL of the porous materials with embedded periodic scatterers in a square duct. The effects of air flow resistivity of the porous materials and number of scatterers on the performance are investigated, and a decoupled analysis is carried out to illustrate the mechanism of the TL improvement.

\section{METHOD AND DESIGN}

Figure 1 shows a duct with a square cross section of $h \times h$, where six identical rigid scatterers are installed inside a block of porous material periodically with a space of $l_{2}$. Each scatterer is $l_{1}$ long, $w_{1}$ wide, and $h$ height, while the porous material block has a length of $l_{3}$.

The TL in the duct can be estimated analytically using the transfer matrix method (Munjal, 2014; Jena et al., 2019), and the transfer matrix of a block of air in a uniform duct without any scatters or porous materials can be expressed by

$$
\mathbf{T}_{\text {air }}=\left[\begin{array}{cc}
\cos \left(k_{0} l\right) & \mathrm{i} \frac{Z_{0} \sin \left(k_{0} l\right)}{S} \\
\mathrm{i} \frac{S \sin \left(k_{0} l\right)}{Z_{0}} & \cos \left(k_{0} l\right)
\end{array}\right],
$$

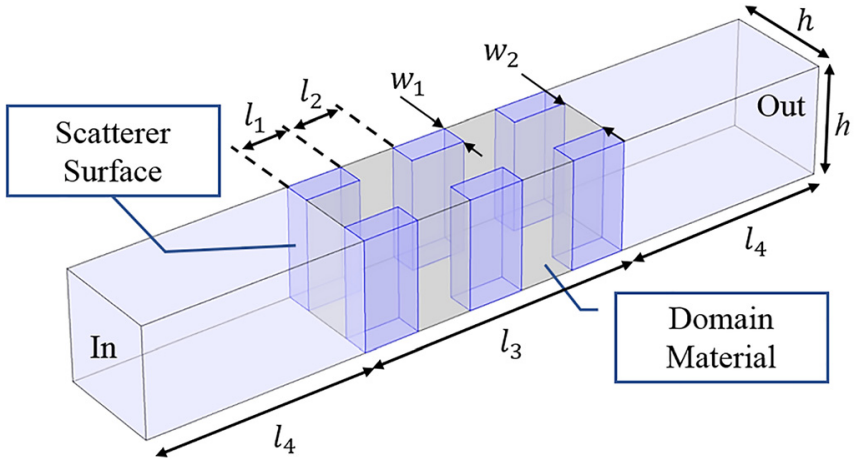

FIG. 1. (Color online) Schematic of the proposed design in a duct with six scatterers.

where $k_{0}=2 \pi f / c_{0}, f$ is the frequency, $c_{0}$ is the speed of sound in air, $l$ is the duct segment length, $i=\sqrt{(-1)}$, $Z_{0}=\rho_{0} c_{0}$ is the characteristic acoustic impedance of air, and $S=h^{2}$ is the duct cross sectional area.

The Delany-Bazley model, instead of more complete and precise models such as the Johnson-Champoux-Allard model (Abbad et al., 2019), is chosen to model the porous material due to the comparative simplicity of the former model over the latter. With the Delany-Bazley model (Allard and Atalla, 2009; Gulia and Gupta, 2018), the complex wave number $\left(k_{\mathrm{c}}\right)$ and complex acoustic impedance $\left(Z_{\mathrm{c}}\right)$ for the porous material can be estimated by

$$
\begin{aligned}
& k_{\mathrm{c}}=k_{0}\left(1+0.098\left(\frac{\rho_{0} f}{R_{f}}\right)^{-0.7}-0.189 \mathrm{i}\left(\frac{\rho_{0} f}{R_{f}}\right)^{-0.595}\right) \\
& Z_{\mathrm{c}}=Z_{0}\left(1+0.057\left(\frac{\rho_{0} f}{R_{f}}\right)^{0.734}-0.087 \mathrm{i}\left(\frac{\rho_{0} f}{R_{f}}\right)^{0.732}\right)
\end{aligned}
$$

where $R_{f}$ is the air flow resistivity of porous material. The transfer matrix of the duct segment filled with the porous material but without any scatters is

$$
\mathbf{T}_{\text {por }}=\left[\begin{array}{cc}
\cos \left(k_{\mathrm{c}} l\right) & \mathrm{i} \frac{Z_{\mathrm{c}} \sin \left(k_{c} l\right)}{S} \\
\mathrm{i} \frac{S \sin \left(k_{\mathrm{c}} l\right)}{Z_{\mathrm{c}}} & \cos \left(k_{\mathrm{c}} l\right)
\end{array}\right] \text {. }
$$

For the arrangement with the periodic arrangement of the scatterers in the duct shown in Fig. 1, the equivalent transfer matrix can be written as

$$
\mathbf{T}=\left[\begin{array}{ll}
T_{11} & T_{12} \\
T_{21} & T_{22}
\end{array}\right]=\mathbf{T}_{\text {small }} \mathbf{T}_{\text {large }} \mathbf{T}_{\text {small }} \mathbf{T}_{\text {large }} \mathbf{T}_{\text {small }}
$$

where the subscript "small" indicates the scatterer section with a small cross-section area and the subscript "large" indicates the intermediate duct section between two scatterers, which has a large cross-section area. When the segment is filled with the air, the corresponding transfer matrices are 


$$
\begin{aligned}
& \mathbf{T}_{\text {small }}=\left[\begin{array}{cc}
\cos \left(k_{0} l_{1}\right) & \frac{\mathrm{i} \sin \left(k_{0} l_{1}\right) Z_{0}}{S_{\text {smal }}} \\
\frac{\mathrm{i} \sin \left(k_{0} l_{1}\right) S_{\text {smal }}}{Z_{0}} & \cos \left(k_{0} l_{1}\right)
\end{array}\right], \\
& \mathbf{T}_{\text {large }}=\left[\begin{array}{cc}
\cos \left(k_{0} l_{2}\right) & \frac{\mathrm{i} \sin \left(k_{0} l_{2}\right) Z_{0}}{S_{\text {large }}} \\
\frac{\mathrm{i} \sin \left(k_{0} l_{2}\right) S_{\text {large }}}{Z_{0}} & \cos \left(k_{0} l_{2}\right)
\end{array}\right],
\end{aligned}
$$

while for the porous medium, they are expressed by

$$
\begin{aligned}
& \mathbf{T}_{\text {small }}=\left[\begin{array}{cc}
\cos \left(k_{\mathrm{c}} l_{1}\right) & \frac{\mathrm{i} \sin \left(k_{\mathrm{c}} l_{1}\right) Z_{\mathrm{c}}}{S_{\text {smal }}} \\
\frac{\mathrm{i} \sin \left(k_{\mathrm{c}} l_{1}\right) S_{\text {smal }}}{Z_{\mathrm{c}}} & \cos \left(k_{\mathrm{c}} l_{1}\right)
\end{array}\right], \\
& \mathbf{T}_{\text {large }}=\left[\begin{array}{cc}
\cos \left(k_{\mathrm{c}} l_{2}\right) & \frac{\mathrm{i} \sin \left(k_{\mathrm{c}} l_{2}\right) Z_{\mathrm{c}}}{S_{\text {large }}} \\
\frac{\mathrm{i} \sin \left(k_{\mathrm{c}} l_{2}\right) S_{\text {large }}}{Z_{\mathrm{c}}} & \cos \left(k_{\mathrm{c}} l_{2}\right)
\end{array}\right],
\end{aligned}
$$

where $S_{\text {small }}$ and $S_{\text {large }}$ are the areas of the ducts with small and large section area, respectively. The sound TL can be estimated using

$$
T L=20 \log _{10}\left|\frac{T_{11}+S T_{12} / Z_{0}+T_{21} Z_{0} / S+T_{22}}{2}\right| .
$$

\section{SIMULATIONS AND DISCUSSIONS}

The acoustic module of COMSOL version 5.4 is used for numerical simulations. The inlet and outlet of the duct

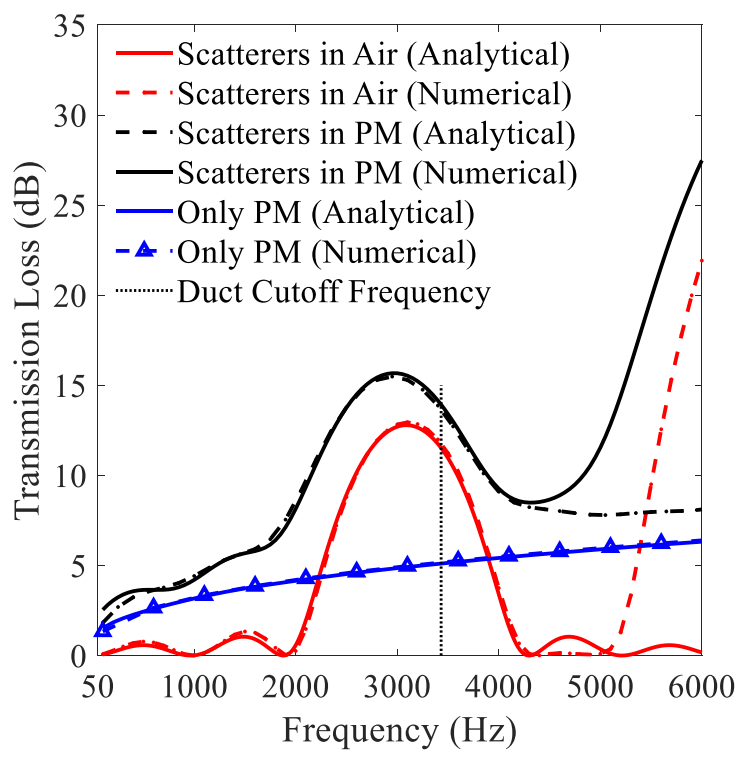

(a) are defined as acoustic ports with a non-reflecting acoustic boundary. The duct walls are defined as acoustically rigid. The upstream and downstream duct segments are air in all simulations. The meshes generated by using tetrahedral elements have the maximum size of $c_{0} /\left(f_{\max } \times 8\right)$, where $c_{0}=344 \mathrm{~m} / \mathrm{s}$ is the sound speed and $f_{\max }=6000 \mathrm{~Hz}$ is the maximum frequency of interest. The density of air is taken as $1.21 \mathrm{~kg} / \mathrm{m}^{3}$. The boundary layer meshing is incorporated on the wall of scatterers. The inlet is excited with a harmonic pressure oscillation with an amplitude of $1.0 \mathrm{~Pa}$, and the frequency resolution in the simulations is $20 \mathrm{~Hz}$. The lossless Helmholtz wave equation has been solved for the numerical estimation (Jena et al., 2019), and the sound TL is estimated by $T L=10 \log _{10}\left(P_{\text {in }} / P_{\text {out }}\right)$, where $P_{\text {in }}$ and $P_{\text {out }}$ are the sound power at inlet and outlet of the duct, respectively. The upstream and downstream segments of the duct contain an air medium of length $l_{4}=100 \mathrm{~mm}$. In the simulations, $l_{1}=l_{2}=25 \mathrm{~mm}, w_{1}=12.5 \mathrm{~mm}, w_{2}=25 \mathrm{~mm}$, and the duct height $h=50 \mathrm{~mm}$. The length of the domain carrying scatterers is $l_{3}=125 \mathrm{~mm}$, and the total duct length under investigation is $\left(2 l_{4}+l_{3}\right)=325 \mathrm{~mm}$. The plane wave cutoff frequency of the duct is $3432 \mathrm{~Hz}$.

Figure 2(a) compares the TL obtained by using the analytical method described in Sec. II and the numerical simulations. The analytical method is only valid below the duct cut-off frequency, where the numerical simulations give the same results. Thus, the numerical simulations are believed to be correct and are used for the investigation in the following simulations.

There is a stop band visible in the figure due to the periodic arrangement of the scatterers. The centre frequency of the stop band is approximately $3100 \mathrm{~Hz}$ following Bragg's law, $B_{f}=n c_{0} \sqrt{\pi} / 4 a$, where $B_{f}$ is the Bragg frequency, $n=1,2,3 \ldots$, and $a=l_{1}+l_{2}$ is the distance between two

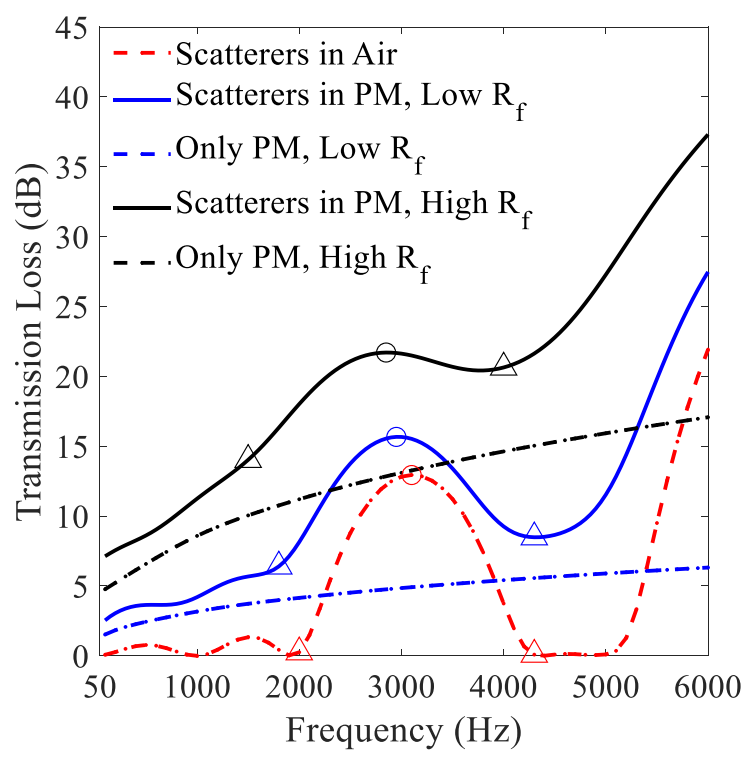

(b)

FIG. 2. (Color online) Transmission loss (a) of the periodic scatterers in the duct when the domain material is air (Air) or the porous medium (PM), and when only the porous material without scatterers is in the duct; (b) of the mixed structure of the scatterers and the porous medium with different airflow resistivity (the low airflow resistivity $R_{f}=1000 \mathrm{~Pa} \mathrm{~s} / \mathrm{m}^{2}$ and the high airflow resistivity $R_{f}=5000 \mathrm{~Pa} \mathrm{~s} / \mathrm{m}^{2}$ ). 


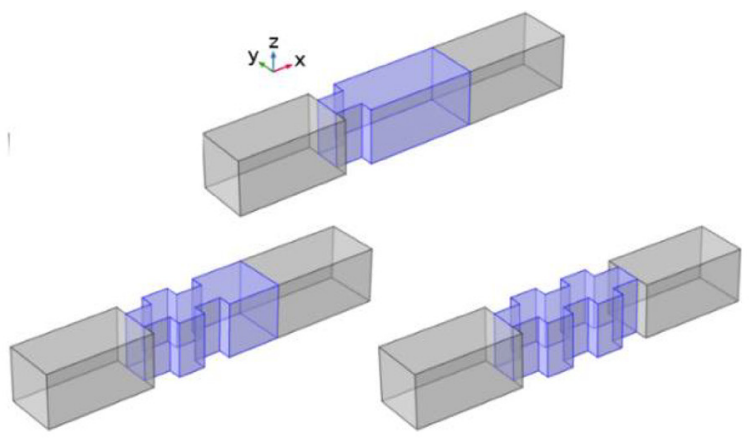

(a)

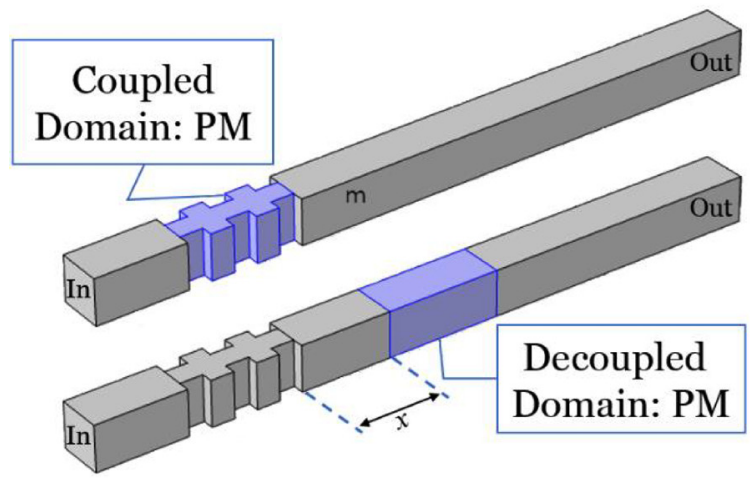

(c)

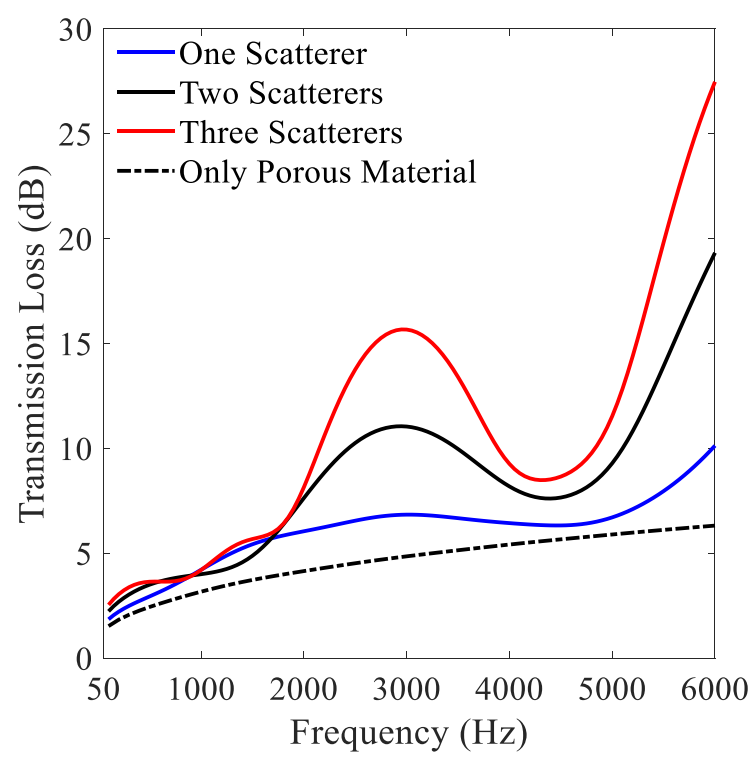

(b)

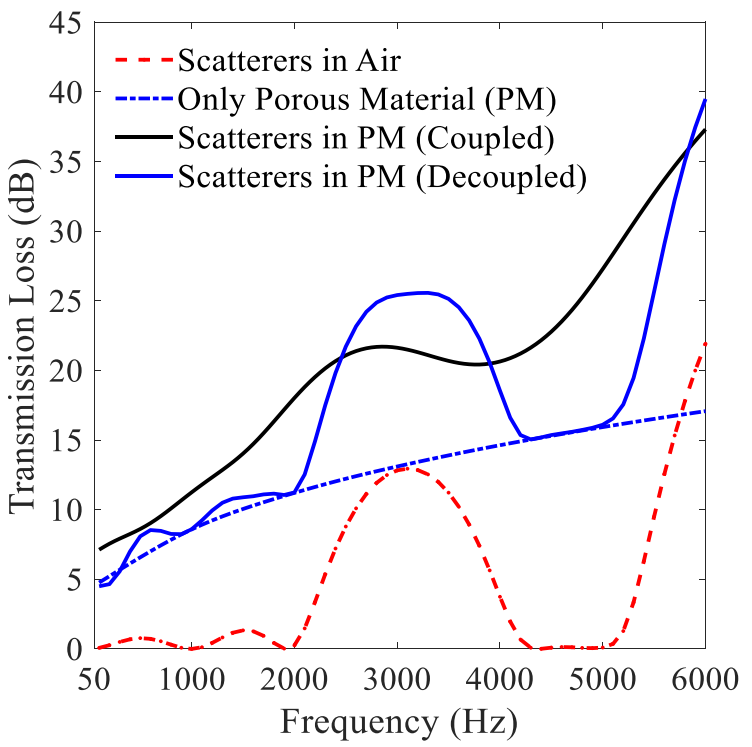

(d)

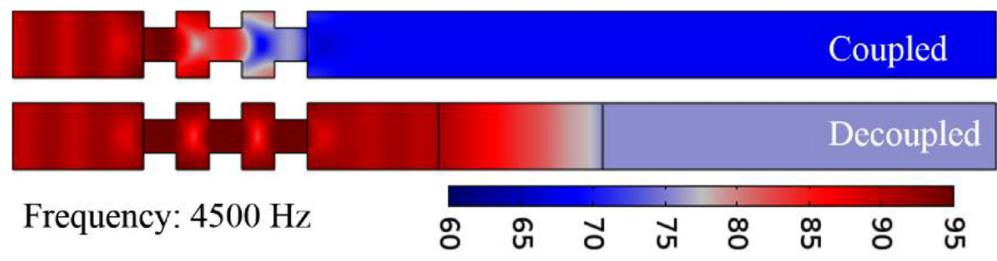

(e)

FIG. 3. (Color online) (a) Three-dimensional (3D) domain with different number of the scatterers in the porous material with $R_{f}=1000$ Pa s/m ${ }^{2}$; (b) the transmission loss corresponding to (a); (c) $3 \mathrm{D}$ domain for coupled and decoupled configurations of the scatterers and porous materials $\left(\mathrm{PM}, R_{f}=5000 \mathrm{~Pa} / \mathrm{m}^{2}\right)$; $(\mathrm{d})$ the transmission loss corresponding to (c); and (e) the distribution of total sound pressure level at $4500 \mathrm{~Hz}$ for the coupled and decoupled configurations.

consecutive scatterers (lattice constant of periodicity). The width and amplitude of the stop band are determined by the geometry of the scatterers and corresponding arrangement (Sharma et al., 2015).
For the TL of the porous material $\left(R_{f}=1000 \mathrm{~Pa} \mathrm{~s} / \mathrm{m}^{2}\right)$ with the scatterers, Fig. 2(a) shows that both the amplitude and bandwidth around the Bragg resonance frequency increase compared to that of the scatterers in air. The airflow 


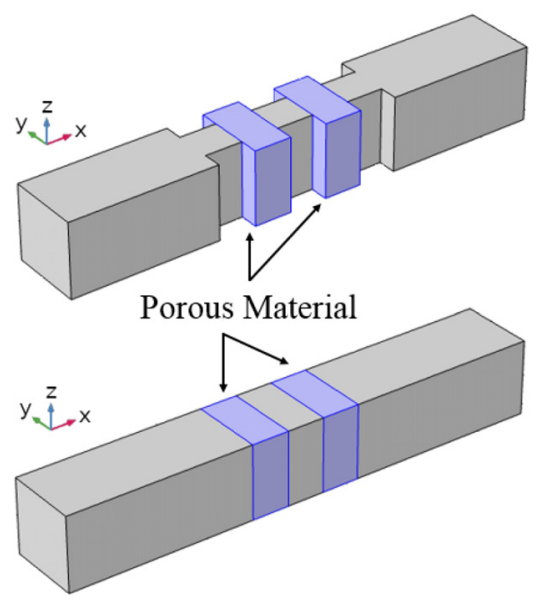

(a)

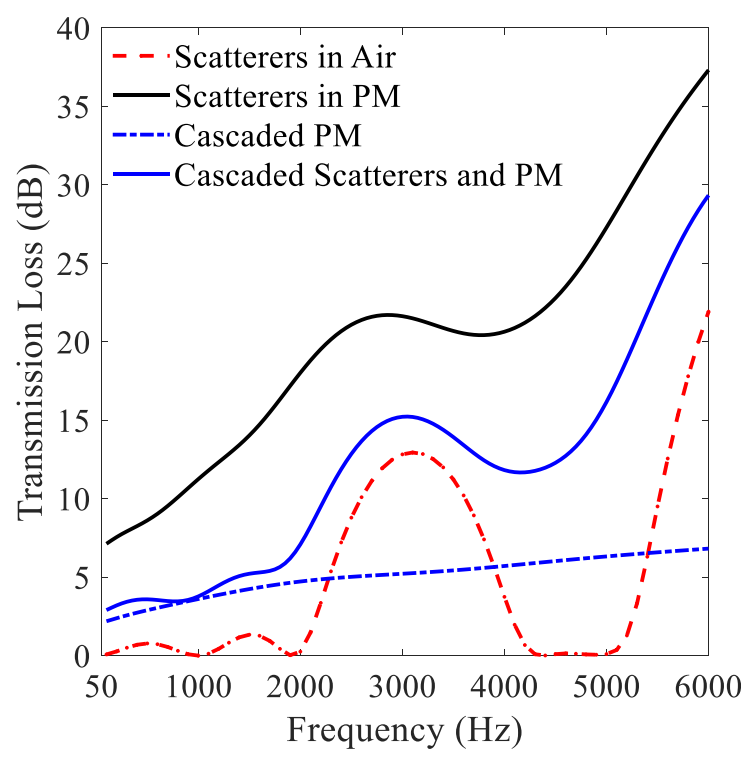

(b)

FIG. 4. (Color online) (a) A Multi-layered configurations of the scatterers and porous materials ( $\left.\mathrm{PM}, R_{f}=5000 \mathrm{~Pa} / \mathrm{m}^{2}\right)$; (b) the corresponding transmission loss.

resistivity of the porous materials affects the TL of the mixed structure, and the simulation results with different airflow resistivities are shown in Fig. 2(b). For comparisons, the TL of the same length of porous material $\left(l_{3}=125 \mathrm{~mm}\right)$ without any scatterers is also shown in the figure.

In the figure, the peak TL around the Bragg resonance occurs at different frequencies with different flow resistivities. The peak values are $12.9,15.8$, and $21.7 \mathrm{~dB}$ for air, the porous materials with the flow resistivity are 1000 and $5000 \mathrm{Pas} / \mathrm{m}^{2}$, respectively, and the corresponding frequencies are 3100,2950 , and $2850 \mathrm{~Hz}$, respectively. The TL peak value becomes larger and the corresponding frequency becomes lower with large airflow resistivity porous materials. The TL in the first pass band increases dramatically after the Bragg resonance frequency, whose value is much larger than the sum of the TLs from the scatterers in air and that from the porous material without the scatterers.

For example, at $4.5 \mathrm{kHz}$, the TL of the mixed structure is approximately $23 \mathrm{~dB}$ with $R_{f}=5000 \mathrm{Pas} / \mathrm{m}^{2}$, while the TLs for the scatterers in air and the porous material without the scatterers at the same frequency are approximately 0.1 and $15 \mathrm{~dB}$, respectively.

The TL of the mixed structure with a different number of scatterers is investigated. Figure 3(a) shows the configurations and Fig. 3(b) shows the results for the porous material with an airflow resistivity of $1000 \mathrm{~Pa} \mathrm{~s} / \mathrm{m}^{2}$. It is clear that when the scatterer number increases, the TL increases around and above the Bragg resonance frequency.

Around and after the Bragg resonance frequency, the significant increase of the TL in the mixed structure is caused by the multiple scattering occurring within the porous materials due to the presence of the periodic scatterers. If the porous materials and the scatterers are widely separated from each other, the "coupling" will not happen.
Figures 3(c) and 3(d) show the configurations and the results where the duct length is changed to $750 \mathrm{~mm}$ so that the porous material $(125 \mathrm{~mm}$ long) and the scatterers can be "decoupled" with a distance of $100 \mathrm{~mm}$. The flow resistivity of the porous material is $R_{f}=5000 \mathrm{~Pa} \mathrm{~s} / \mathrm{m}^{2}$. It is clear that the TL of the mixed structure in the decoupled configuration is the sum of that of the porous material and the scatterers, while in the coupled configuration, the scatterers reduce the $\mathrm{TL}$ of the mixed structure around the Bragg resonance frequency while increasing it at the other frequencies. Figure 3(e) shows the corresponding sound pressure level distribution at $4500 \mathrm{~Hz}$, where the sound pressure decreases due to scattering between the scatterers within the porous medium.

In practical implementations, it might be easier to have the porous materials and the scatterers installed separately in different layers. Figure 4(a) shows a multi-layered configuration with cascaded scatterers and the porous material panels, where the difference with the previous configurations is that there is air instead of the porous materials in the small cross section formed by the scatterers in the duct. The results are shown in Fig. 4(b). Although putting the scatterers in the porous materials gives the highest TL, this multilayered configuration can provide moderate TL, which is still much larger than the sum of the TL from the scatterers in air and that from the porous material without the scatterers.

\section{CONCLUSION}

In the present work, the acoustically rigid scatterers are installed periodically in a porous material in a square duct to increase the TL of the porous material. The Delany-Bazley acoustic impedance model is used to model the porous material and the transfer matrix method is used to calculate the 
TL of the mixed structure in the duct under the cut-off frequency of the duct to verify the numerical simulations. It is found that TL can be improved significantly with this mixed structure. The effects with different air flow resistivities of the porous materials and numbers of scatterers are investigated, and the decoupled simulations reveal that the TL of the mixed structure is larger than the sum of the TL from the individual components at frequencies larger than the first Bragg resonance. This improvement is due to scattering between the scatterers within the porous medium. A practical multi-layered configuration with cascaded scatterers and porous material panels is proposed, which can also provide larger TL than the sum of the TLs of the individual components. Future work includes carrying out experiments to demonstrate the potential of mixed structure in broadband sound attenuation and designing of scatterers to enhance the attenuation in the low frequency range.

\section{ACKNOWLEDGMENTS}

D.P.J. wants to acknowledge NIT Rourkela for permitting him to visit the Centre for Audio, Acoustics and Vibration (CAAV), University of Technology Sydney to carry out the reported research.

Aa, B. V. D., and Forssén, J. (2014). "Scattering by an array of perforated cylinders with a porous core," J. Acoust. Soc. Am. 136(5), 2370-2380.

Abbad, A., Atalla, N., Ouisse, M., and Doutres, O. (2019). "Numerical and experimental investigations on the acoustic performances of membraned Helmholtz resonators embedded in a porous matrix,” J. Sound. Vib. 459, 114873 .

Allard, J. F., and Atalla, N. (2009). Propagation of Sound in Porous Media: Modelling Sound Absorbing Materials, 2nd ed. (John Wiley \& Sons, New York).

Doutres, O., Atalla, N., and Osman, H. (2015). "Transfer matrix modeling and experimental validation of cellular porous material with resonant inclusions," J. Acoust. Soc. Am. 137(6), 3502-3513.

Groby, J.-P., Lagarrigue, C., Brouard, B., Dazel, O., Tournat, V., and Nennig, B. (2014). "Using simple shape three-dimensional rigid inclusions to enhance porous layer absorption," J. Acoust. Soc. Am. 136(3), $1139-1148$.
Gulia, P., and Gupta, A. (2018). "Enhancing the sound transmission loss through acoustic double panel using sonic crystal and porous material," J. Acoust. Soc. Am. 144, 1435-1442.

Gupta, A., Lim, K. M., and Chew, C. H. (2012). "Parametric study on rectangular sonic crystal,” Appl. Mech. Mater. 152-154, 281-286.

Jena, D. P., Dandsena, J., and Jayakumari, V. G. (2019). "Demonstration of effective acoustic properties of different configurations of Helmholtz resonators," Appl. Acoust. 155, 371-382.

Kang, Y. J., and Bolton, J. S. (1996). "A finite element model for sound transmission through foam-lined double-panel structures," J. Acoust. Soc. Am. 99(5), 2755-2765.

Lagarrigue, C., Groby, J. P., Tournat, V., Dazel, O., and Umnova, O. (2013). "Absorption of sound by porous layers with embedded periodic arrays of resonant inclusions," J. Acoust. Soc. Am. 134(6), 4670-4680.

Leclaire, P., Umnova, O., Dupont, T., and Panneton, R. (2015). “Acoustical properties of air-saturated porous material with periodically distributed dead-end pores," J. Acoust. Soc. Am. 137(4), 1772-1782.

Munjal, M. L. (2014). Acoustics of Ducts and Mufflers, 2nd ed. (John Wiley \& Sons, New York).

Olny, X., and Boutin, C. (2003). "Acoustic wave propagation in double porosity media,” J. Acoust. Soc. Am. 114(1), 73-89.

Panneton, R., and Atalla, N. (1996). "Numerical prediction of sound transmission through finite multilayer systems with poroelastic materials," J. Acoust. Soc. Am. 100(1), 346-354.

Pieren, R., and Heutschi, K. (2015). "Modelling parallel assemblies of porous materials using the equivalent circuit method," J. Acoust. Soc. Am. 137(2), EL131-EL136.

Roh, H.-S., and Yoon, S. W. (2004). "Acoustic diagnosis for porous medium with circular cylindrical pores,” J. Acoust. Soc. Am. 115(3), 1114-1124.

Sánchez-Dehesa, J., García-Chocano, V. M., Torrent, D., Cervera, F., Cabrera, S., and Simon, F. (2011). "Noise control by sonic crystal barriers made of recycled materials," J. Acoust. Soc. Am. 129(3), 1173-1183.

Sanchez-Perez, J. V., Rubio, C., Martinez-Sala, R., Sanchez-Grandia, R., and Gomez, V. (2002). "Acoustic barriers based on periodic arrays of scatterers," Appl. Phys. Lett. 81(27), 5240-5242.

Sharma, G. S., Eggler, D., Peters, H., Kessissoglou, N., Skvortsov, A., and MacGillivray, I. (2015). "Acoustic performance of periodic composite materials," in Proceedings of the Australian Acoustical Society, Acoustics 2015, November 15-18, Hunter Valley, Australia.

Song, B. H., and Bolton, J. S. (2000). “A transfer-matrix approach for estimating the characteristic impedance and wave numbers of limp and rigid porous materials," J. Acoust. Soc. Am. 107(3), 1131-1152.

Takahashi, D. (1989). "Excess sound absorption due to periodically arranged absorptive materials," J. Acoust. Soc. Am. 86(6), 2215-2222.

Tang, S. K. (2018). "Improving traffic noise transmission loss of plenum windows by using sound scatterer arrays," J. Acoust. Soc. Am. 143, 1715.

Utley, W. A., and Mulholland, K. A. (1968). "The transmission loss of double and triple walls," Appl. Acoust. 1(1), 15-20. 